Proceedings of the 16th Czech and Slovak Conference on Magnetism, Košice, Slovakia, June 13-17, 2016

\title{
DC NanoSQUID from Nb Thin Films
}

\begin{abstract}
V. ŠTrbík*, M. PisArČíK, Š. GaŽI And M. ŠPAnková
Institute of Electrical Engineering, Slovak Academy of Sciences, Dúbravská cesta 9, 84104 Bratislava, Slovakia

We have prepared $\mathrm{Nb}$ thin film (thickness 80-100 nm) nanoSQUID with two symmetrically placed nanosized constrictions in superconducting loop. The nanoSQUID was prepared in two steps: at first a microsized SQUID was prepared and in the second step nanosized constrictions of the width of $120 \mathrm{~nm}$ in the SQUID loop were created by a focused ion beam technique. We studied the electrical and magnetic properties. From the obtained results, SQUID and constriction dimensions, the spin sensitivity was estimated to be about $50 \mu_{\mathrm{B}} \mathrm{Hz}^{-1 / 2}$. Further improvement of spin sensitivity is possible by lowering the SQUID and constriction dimensions.
\end{abstract}

DOI: 10.12693/APhysPolA.131.1054

PACS/topics: 74.78.--w, 74.70.Ad, 85.25.Dq

\section{Introduction}

Superconducting quantum interference device (SQUID) is able to detect with an ultrahigh sensitivity a magnetic flux or any physical quantity that can be converted to a magnetic flux threading the SQUID loop [1]. The idea of reducing the SQUID size to improve its inductive coupling to small magnetic particles (magnetic nanoobjects) was introduced by Ketchen et al. [2]. Their considerations showed that SQUID capability to detect the magnetic moment is proportional to a diameter of its loop and the sensitivity of miniaturized SQUID can be expressed in number of spins or in units of the Bohr magneton $\mu_{\mathrm{B}}$. The authors $[3,4]$ developed the nanoSQUIDs for the detection of small spin populations, having a hole with size of $200 \mathrm{~nm}$ and resulting spin sensitivities $250 \mathrm{spin} / \sqrt{ } \mathrm{Hz}[3]$ or $65 \mu_{\mathrm{B}} / \sqrt{ } \mathrm{Hz}$ [4]. Recently, Pb-based nanoSQUID (hole diameter of $46 \mathrm{~nm}$ ) has been presented with ultra-high spin sensitivity of about $0.4 \mu_{\mathrm{B}} / \sqrt{ } \mathrm{Hz}$ [5]. High magnetic moment sensitivity of SQUID can also be obtained using the SQUID Josephson junctions with a very small cross-section. Thus, the nanoSQUID can have either submicron loop or nanometric Josephson junctions. In this paper we present the properties of Nb-based nanoSQUIDs with two nanosized constrictions of appropriate magnetic moment sensitivity.

\section{Experimental details}

The nanoSQUID was prepared in two steps. In the first step Nb-based microSQUID was created, in the second step using focused ion beam (FIB) technique two nanobridges of width 120-270 nm were patterned symmetrically in the SQUID loop (Fig. 1). The microSQUID was prepared from two Nb layers separated by $\mathrm{Al} / \mathrm{AlO}_{x}$ barrier which limits the superconducting current in SQUID loop to level about $100 \mu \mathrm{A}$ and ensures

*corresponding author; e-mail: elekstrb@savba.sk a non-hysteresis behaviour of current-voltage characteristic. The Nb1 layer $(100 \mathrm{~nm})$ was deposited by dc magnetron sputtering on $\mathrm{Si}$ (001) substrate and patterned by wet etching. The Nb2 layer $(80 \mathrm{~nm})$ was deposited again by dc magnetron sputtering but patterned by a lift-off technique. The FIB patterning was performed on Quanta 3D 200i (FEI company) equipment. Electrical and magnetic properties of SQUIDs were measured using 4-point dc method in an applied magnetic field.
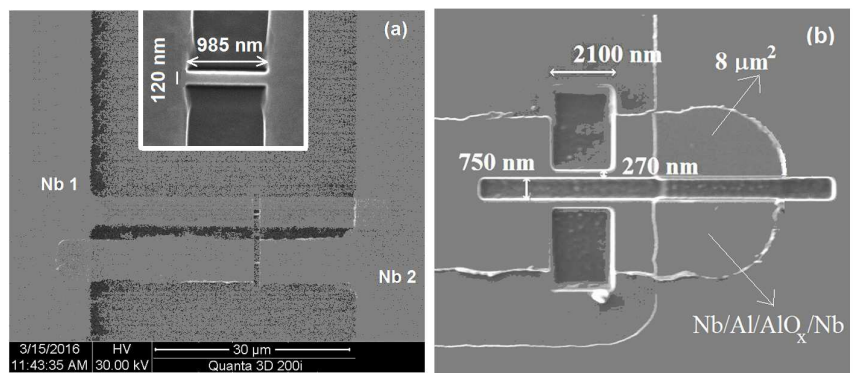

Fig. 1. The measured nanoSQUIDs based on nanosized constrictions in SQUID loop. The width of constriction is $120 \mathrm{~nm}$ (a) and $270 \mathrm{~nm}$ (b).

\section{Results}

Both $\mathrm{Nb}$ films exhibit partially reduced critical temperatures $(\mathrm{Nb} 1 \approx 8.4 \mathrm{~K}, \mathrm{Nb} 2 \approx 8 \mathrm{~K})$ and after FIB patterning of nanosized constrictions an addional resistance tail in resistance vs. temperature $(R(T))$ dependence was measured (Fig. 2). Despite the suppressed superconductivity the working temperature of SQUID structure $(4.2 \mathrm{~K})$ is sufficiently low for reliable performance of the SQUID. The inset in Fig. 2 shows the SQUID temperature dependence of resistance from room temperature down to $4.2 \mathrm{~K}$.

Typical current-voltage characteristics $(\mathrm{CVCH})$ of nanoSQUID for various magnetic field taken at $4.2 \mathrm{~K}$ are shown in Fig. 3. The maximum critical current of about $100 \mu \mathrm{A}$ and non-hysteretic behavior of $\mathrm{CVCH}$ were obtained after adjustment of $\mathrm{Al} / \mathrm{AlO}_{x}$ barrier thickness between the Nb layers. Therefore we do not need to shunt the SQUID. 


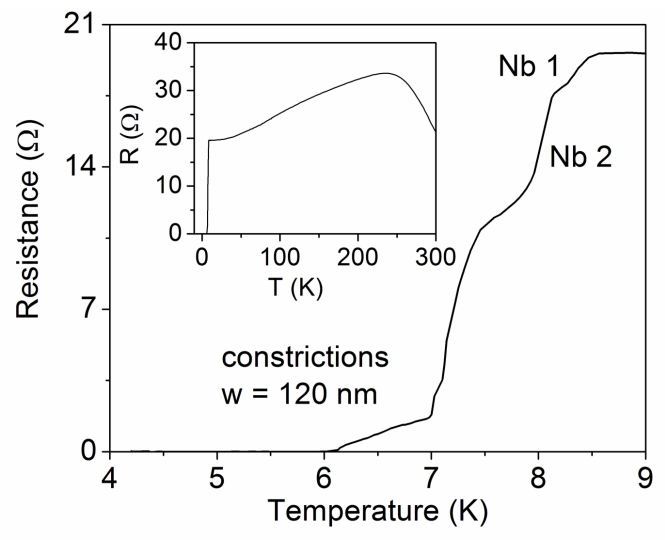

Fig. 2. The temperature dependence of nanoSQUID resistance. The superconducting transitions of individual layers $\mathrm{Nb} 1, \mathrm{Nb} 2$ and two constrictions are visible. Inset shows the dependence in temperature range 4$300 \mathrm{~K}$.

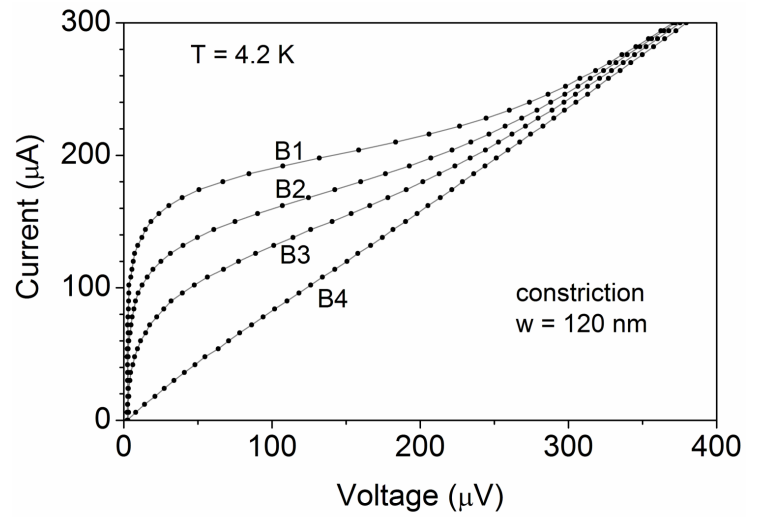

Fig. 3. The current-voltage characteristics of nanoSQUID with constrictions $(w=120 \mathrm{~nm})$ for various magnetic field.

The dependences of SQUID voltage on applied magnetic field $(U(B))$ at various bias current are shown in Fig. 4. One can see that the optimal bias current for the largest voltage response to magnetic field is around $160 \mu \mathrm{A}$. At a current of 100 or $200 \mu \mathrm{A}$ the SQUID sensitivity field strongly decreases. The highest voltage response to field $(55 \mathrm{~V} / \mathrm{T})$ was observed at current of $162 \mu \mathrm{A}$. This value is important to estimate a spin sensitivity of SQUID (in number of spins) which is defined as $S_{n}=2 a \Phi_{n s} / \mu_{0} \mu_{\mathrm{B}}$ or $S_{n}=4 w \Phi_{n s} / \alpha \mu_{0} \mu_{\mathrm{B}}$ where $a$ is a loop radius, $\Phi_{n s}$ is the SQUID flux noise, $w$ is the width of constriction, $\mu_{0}$ is vacuum permeability, $\mu_{\mathrm{B}}$ is the Bohr magneton and $\alpha$ is coupling factor. In the second formula the loop radius $a$ was replaced by the effective geometrical parameter $2 w / \alpha[6]$.

From the known sensitivity $(55 \mathrm{~V} / \mathrm{T})$, SQUID area and standard voltage resolution of electronic equipment $\Delta U \approx 1 \mathrm{nV} \mathrm{Hz}^{-1 / 2}$ we can estimate the flux noise $\Phi_{n s}=2.45 \times 10^{-21} \mathrm{~Wb} \mathrm{~Hz}^{-1 / 2}$. Compared with the

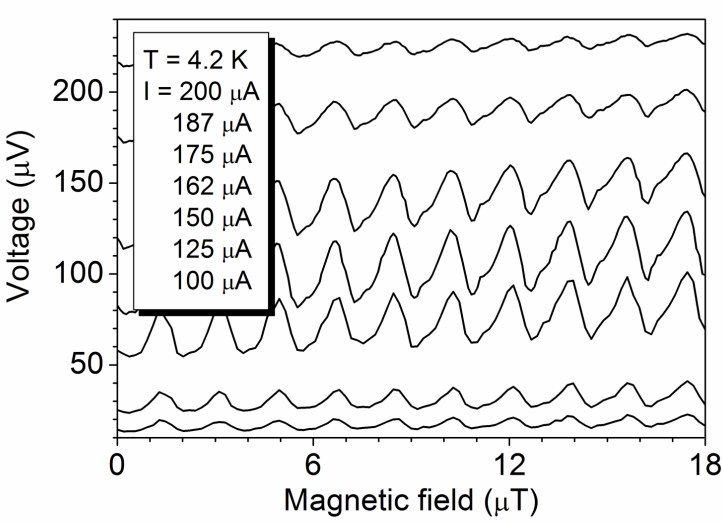

Fig. 4. The dependences of SQUID voltage on applied magnetic field for various bias current at temperature of $4.2 \mathrm{~K}$. The maximal sensitivity $(55 \mathrm{~V} / \mathrm{T})$ was obtained at bias current of $162 \mu \mathrm{A}$.

value of magnetic flux quantum $\left(\Phi_{0}=2.07 \times 10^{-15} \mathrm{~Wb}\right)$ the flux noise reaches $\Phi_{n s}=1.19 \times 10^{-6} \Phi_{0}$ which is a typical value of flux noise of SQIUDs [7]. Considering $w=120 \mathrm{~nm}$ and ideal coupling limit $\alpha=0.5$ (Fig. 5) we obtain the spin sensitivity (in number of spins) of $202 \mu_{\mathrm{B}} \mathrm{Hz}^{-1 / 2}$. The real value of coupling factor $\alpha$ will be lower. The width $w=120 \mathrm{~nm}$ of constriction is comparable to the London penetration depth $\lambda_{\mathrm{L}}$. Gubin et al. [8] estimated $\lambda_{\mathrm{L}}(0) \approx 100 \mathrm{~nm}$ for $\mathrm{Nb}$ films 80-100 $\mathrm{nm}$ thick. Considering the temperature dependence of $\lambda_{\mathrm{L}}$, the screening ability of constriction is significantly decreased and we estimate that about $45 \%$ magnetic flux is penetrated into loop of SQUID corresponding to $\alpha \approx 0.2$. In this case the spin sensitivity decreases to about $500 \mu_{\mathrm{B}} \mathrm{Hz}^{-1 / 2}$. The further optimization of SQUID area and narrowing of constrictions can lead to improved spin sensitivity of the prepared nanoSQUIDs.



Fig. 5. Schematics showing the coupling of magnetic dipole to the SQUID in place of nanosized constriction. When half of the flux lines are threaded by the constriction, the ideal coupling limit of 0.5 is achieved.

\section{Conclusions}

We presented the possibility to prepare the nanoSQUID with two constrictions of width $120 \mathrm{~nm}$ 
symmetrically placed in SQUID loop. At first the microsized SQUID was created by a standard photolithography and wet etching and then by focused ion beam technique the nanosized constrictions were patterned. The current-voltage characteristics exhibited a critical current of about $100 \mu \mathrm{A}$ and a non-hysteretic behaviour. From voltage vs. magnetic flux dependences the flux noise sensitivity $\Phi_{n s}=1.19 \times 10^{-6} \Phi_{0}$ was determined.

\section{Acknowledgments}

This work was supported by the Slovak Research and Development Agency, APVV-0494-11, Slovak Grant Agency for Science, VEGA 2/0120/14, by Structural Funds of the European Union by means of the Agency of the Ministry of Education, Science, Research and Sport of the Slovak Republic in the projects "CENTE II" ITMS code 26240120019. The authors acknowledge the fruitful collaboration within COST Action MP1308 TO-BE.

\section{References}

[1] J. Clarke, IEEE Trans. Electron Dev. 27, 1896 (1980).

[2] M.B. Ketchen, D.D. Awschalom, W.J. Gallagher, A.W. Kleinsasser, R.L. Sandstrom, J.R. Rozen, B. Bumble, IEEE Trans. Magn. 25, 1212 (1989).

[3] S.K.H. Lam, D.L. Tilbrook, Appl. Phys. Lett. 82, 1078 (2003).

[4] A. Finkler, Y. Segev, Y. Myasoedov, M.L. Rappaport, L. Ne'eman, D. Vasyukov, E. Zeldov, M.E. Huber, J. Martin, A. Yacoby, Nano Lett. 10, 1046 (2010).

[5] D. Vasyukov, Y. Anahory, L. Embon, D. Halbertal, J. Cuppens, L. Ne'eman, A. Finkler, Y. Segev, Y. Myasoedov, M.L. Rappaport, M.E. Huber, E. Zeldov, Nature Nanotechnol. 8, 639 (2013).

[6] V. Bouchiat, Supercond. Sci. Technol. 22, 064002 (2009).

[7] D.L. Tilbrook, Supercond. Sci. Technol. 22, 064003 (2009).

[8] A.I. Gubin, K.S. Il'in, S.A. Vitusevich, M. Siegel, N. Klein, Phys. Rev. B 72, 064503 (2005). 